\title{
EXPLICIT SOLUTION FOR AN INFINITE DIMENSIONAL GENERALIZED INVERSE EIGENVALUE PROBLEM
}

\author{
KAZEM GHANBARI
}

(Received 28 March 2000 and in revised form 7 September 2000)

\begin{abstract}
We study a generalized inverse eigenvalue problem (GIEP), $A x=\lambda B x$, in which $A$ is a semi-infinite Jacobi matrix with positive off-diagonal entries $c_{i}>0$, and $B=$ $\operatorname{diag}\left(b_{0}, b_{1}, \ldots\right)$, where $b_{i} \neq 0$ for $i=0,1, \ldots$ We give an explicit solution by establishing an appropriate spectral function with respect to a given set of spectral data.
\end{abstract}

2000 Mathematics Subject Classification. 65F18, 47B36.

1. Introduction. Inverse eigenvalue problems, that concern the reconstruction of matrices from a prescribed set of spectral data, are very important subclass of inverse problems that arise in mathematical modeling. There have been challenging work in this area in the last 20 years. Inverse eigenvalue problems mostly are connected to the theory of orthogonal polynomials. An inverse eigenvalue problem appears to be more challenging whence the objective matrix is a specifically structured matrix. Jacobi matrices are among those interesting structured matrices concerned with three term recurrence relations. In this paper we study the generalized inverse eigenvalue problem $A x=\lambda B x$, in which $A$ is a semi-infinite Jacobi matrix of the form

$$
A=\left(\begin{array}{cccccc}
a_{0} & c_{0} & 0 & \ldots & \ldots & \ldots \\
c_{0} & a_{1} & c_{1} & 0 & \ldots & \ldots \\
0 & c_{1} & a_{2} & c_{2} & 0 & \ldots \\
\vdots & \vdots & \vdots & \vdots & \vdots & \vdots
\end{array}\right),
$$

$B=\operatorname{diag}\left(b_{0}, b_{1}, \ldots\right)$, where $b_{i} \neq 0$, and $c_{i}>0$, for $i=0,1, \ldots$ Using the definition of positive definite sequence given by Ahiezer and Krein [1], we establish an explicit formula for recovering the Jacobi matrix $A$ via a given set of spectral data and a diagonal matrix $B$. We use the usual approach of dealing with this type of problems, that is, the orthogonal polynomials. In this paper, we only discuss the infinite dimensional version of GIEP. The finite dimensional case of this problem has been studied by many authors, see for example [4] for the classical case $A x=\lambda x$, and [2, 3] for the generalized case $A x=\lambda B x$.

2. Three term recurrence relation and spectral function. Let $y_{n}(\lambda)$ be a solution of

$$
\begin{gathered}
c_{n} y_{n+1}(\lambda)=\left(b_{n} \lambda-a_{n}\right) y_{n}(\lambda)-c_{n-1} y_{n-1}(\lambda), \quad n=0,1, \ldots, \\
y_{-1}(\lambda)=0, \quad c_{-1} y_{0}(\lambda)=1,
\end{gathered}
$$


where $\left\{a_{n}\right\}_{n \geq 0}$ is an arbitrary sequence of real numbers, $\left\{\boldsymbol{b}_{n}\right\}_{n \geq 0}$, and $\left\{c_{n}\right\}_{n \geq 0}$ are sequences of nonzero real numbers with $c_{n}>0$, for $n=0,1, \ldots$. A complex number $\lambda$ is said to be an eigenvalue of the recurrence relation (2.1) if there is a nonzero solution $y(\lambda)=\left(y_{0}(\lambda), y_{1}(\lambda), \ldots\right)$ satisfying the recurrence relation (2.1). In this case $y(\lambda)=\left(y_{0}(\lambda), y_{1}(\lambda), \ldots\right)^{T}$ is the eigenvector corresponding to $\lambda$. If $\lambda_{r}$ is an eigenvalue and $y^{(r)}=\left(y_{0}\left(\lambda_{r}\right), y_{1}\left(\lambda_{r}\right), \ldots\right)^{T}$ is the corresponding eigenvector, then the number

$$
\rho_{r}=\left\{\sum_{n=0}^{\infty} b_{n}\left|y_{n}\left(\lambda_{r}\right)\right|^{2}\right\}
$$

is called the normalization constant. The set of all eigenvalues and normalization constants of the recurrence relation (2.1) are denoted by $\sigma(A, B)$ and $\rho(A, B)$, respectively.

DEFINITION 2.1. A spectral function $\tau(\lambda)$ associated with the recurrence formula (2.1) is to be nondecreasing, right continuous, satisfying the boundedness requirement

$$
\int_{-\infty}^{\infty} \lambda^{2 n} d \tau(\lambda)<\infty, \quad n=0,1, \ldots
$$

for all $n$, and the orthogonality

$$
\int_{-\infty}^{\infty} y_{p}(\lambda) y_{q}(\lambda) d \tau(\lambda)=b_{p}^{-1} \delta_{p q}, \quad p, q=0,1, \ldots
$$

THEOREM 2.2. There is at least one spectral function for the recurrence relation (2.1).

Proof. See [2, Theorem 5.2.1].

THEOREM 2.3. For every $\lambda$, the recurrence relation (2.1) has at least one nontrivial solution of summable square, in the sense that,

$$
\sum_{0}^{\infty} b_{n}\left|y_{n}(\lambda)\right|^{2}<\infty
$$

Proof. See [2, Theorems 5.4.2, 5.6.1].

DEFINITION 2.4. Let $\left\{y_{n}(\lambda)\right\}_{n \geq 0}$ be an arbitrary solution of the recurrence relation (2.1). If

$$
\sum_{0}^{\infty} b_{n}\left|y_{n}(\lambda)\right|^{2}<\infty
$$

for some $\lambda \in \mathbb{C}$, then we say that the limit-circle case holds for the recurrence relation (2.1).

THEOREM 2.5. Suppose that the limit circle case holds for one value $\lambda=\lambda_{0} \in \mathbb{C}$. Then this is true for all $\lambda \in \mathbb{C}$. 
Proof. See [2, Theorem 5.6.1].

3. Eigenvalues in the limit-circle case. Assuming the limit-circle case to hold, we take it all solutions of (2.1) are summable square in the sense of Theorem 2.3, and uniformly so in any finite $\lambda$-region. We therefore have that

$$
\sum_{0}^{\infty} b_{n}\left|y_{n}(\lambda)\right|^{2}<\eta(\lambda)
$$

where $\eta(\lambda)$ is some function which is bounded for bounded $\lambda$. Adopting some fixed real $\mu$ as an eigenvalue, we define the eigenvalues as the zeros of

$$
(\lambda-\mu) \sum_{0}^{\infty} b_{n} y_{n}(\lambda) y_{n}(\mu)
$$

By (3.1), the function (3.2) is an entire function of $\lambda$. It does not vanish identically, since its derivative is not zero when $\lambda=\mu$. Hence its zeros will have no finite limit. Moreover, these zeros will be real, being the limits of the zeros

$$
(\lambda-\mu) \sum_{0}^{m} b_{n} y_{n}(\lambda) y_{n}(\mu)=c_{m-1}\left\{y_{m}(\lambda) y_{m-1}(\mu)-y_{m}(\mu) y_{m-1}(\lambda)\right\},
$$

the zeros of the later are real [1, Theorem 4.3.1], and the zeros of (3.2) are the limits of the zeros of (3.3), by Rouche's theorem.

Having defined the eigenvalues $\lambda_{r}$ as the roots of

$$
\lim _{m \rightarrow \infty} c_{m-1}\left\{y_{m}(\lambda) y_{m-1}(\mu)-y_{m}(\mu) y_{m-1}(\lambda)\right\}=0,
$$

we may define as a spectral function a step function whose jumps are at the $\lambda_{r}$ and are of amount

$$
\frac{1}{\rho_{r}}=\left\{\sum_{0}^{\infty} b_{n}\left|y_{n}\left(\lambda_{r}\right)\right|^{2}\right\}^{-1}
$$

More formally, we can express this spectral function as follows

$$
\tau(\lambda)=\sum_{\lambda_{r} \leq \lambda} \frac{1}{\rho_{r}}
$$

REMARK 3.1. In general, we may have continuous spectrum for the recurrence relation (2.1). If we consider the discrete Schrödinger operator

$$
H y_{n}=y_{n+1}+y_{n-1}+a_{n} y_{n},
$$

where, $\lim \left|a_{n}\right|=0$, as $n \rightarrow \infty$, then $H$ has continuous spectrum (cf. [5]). 
REMARK 3.2. Note that we can display the recurrence relation (2.1) as

$$
A y=\lambda B y
$$

where

$$
\begin{gathered}
A=\left(\begin{array}{cccccc}
a_{0} & c_{0} & 0 & \ldots & \ldots & \ldots \\
c_{0} & a_{1} & c_{1} & 0 & \ldots & \ldots \\
0 & c_{1} & a_{2} & c_{2} & 0 & \ldots \\
\vdots & \vdots & \vdots & \vdots & \vdots & \vdots
\end{array}\right) \\
B=\operatorname{diag}\left(b_{0}, b_{1}, \ldots\right) \\
y(\lambda)=\left(y_{0}(\lambda), y_{1}(\lambda), \ldots\right)^{T}
\end{gathered}
$$

LEMMA 3.3. Let $A$ and $B$ be the matrices given by (3.9) and (3.10), and let $y(\lambda)$ be the vector given by (3.11). Let $\left\{\lambda_{r}\right\}_{r \geq 0}$ and $\left\{\rho_{r}\right\}_{r \geq 0}$ be the sequence of eigenvalues and the normalization constants, respectively. Then

(i) If $i \neq j$ then $y\left(\lambda_{i}\right)$ is orthogonal to $y\left(\lambda_{j}\right)$ in the sense that

$$
\sum_{p=0}^{\infty} b_{p} y_{p}\left(\lambda_{i}\right) y_{p}\left(\lambda_{j}\right)=\rho_{i} \delta_{i j}
$$

(ii) If $p, q \geq 0$ then

$$
\sum_{r=0}^{\infty} y_{p}\left(\lambda_{r}\right) y_{q}\left(\lambda_{r}\right) \rho_{r}^{-1}=b_{p}^{-1} \delta_{p q} .
$$

The property (ii) is called the dual orthogonality.

Proof. See [2, page 133].

\section{Infinite dimensional GIEP}

Problem 4.1. Let $B=\operatorname{diag}\left(b_{0}, b_{1}, \ldots\right)$ be a given diagonal real matrix, where $b_{i} \neq 0$ for $i=0,1, \ldots$, and let $\left\{\lambda_{i}\right\}$ and $\left\{\rho_{i}\right\}$ be real numbers such that $\lambda_{i} \rho_{i}>0$, for $i=0,1, \ldots$. We find a positive definite semi-infinite Jacobi matrix $A$ that satisfies (3.8) with

(i) $\sigma(A, B)=\left\{\lambda_{0}, \lambda_{1}, \ldots\right\}$,

(ii) $\rho(A, B)=\left\{\rho_{0}, \rho_{1}, \ldots\right\}$.

We call Problem 4.1 infinite dimensional GIEP. It is easy to see that if there is a solution for GIEP then $\lambda_{i} \rho_{i}>0$, for $i=0,1, \ldots$. For, if $\lambda_{i} \in \sigma(A, B)$, and $y^{(i)}$ is the corresponding eigenvector, then

$$
0<y^{(i)^{T}} A y^{i}=\lambda_{i} y^{(i)^{T}} B y^{(i)}=\lambda_{i} \rho_{i}, \quad \text { for } i=0,1, \ldots
$$


LEMMA 4.2. Equation (2.4) with $p \neq q$ is equivalent to

$$
\int_{-\infty}^{\infty} y_{p}(\lambda) \lambda^{q} d \tau(\lambda)=0, \quad 0 \leq q \leq p-1
$$

Proof. See [2, Theorem 4.6.1].

DEFINITION 4.3. Let $\tau(\lambda)$ be the spectral function defined by (3.6). The scalars

$$
\mu_{j}=\int_{-\infty}^{\infty} \lambda^{j} d \tau(\lambda), \quad j=0,1, \ldots
$$

are called the moments of $\tau(\lambda)$.

Note that formula (4.3) is equivalent to

$$
\mu_{j}=\sum_{0}^{\infty} \frac{\lambda_{r}^{j}}{\rho_{r}}, \quad j=0,1, \ldots,
$$

and since $\lambda_{r} \rho_{r}>0$, the odd moments $\mu_{2 n+1}$ are all positive.

Definition 4.4. Let $\tau(\lambda)$ be the spectral function defined by (3.6), and let $\mu_{j}$ be the moments of $\tau(\lambda)$ given by (4.4). We define $M_{n}$ and $\Delta_{n}$ by

$$
\begin{gathered}
M_{n}=\left(\begin{array}{cccc}
\mu_{0} & \mu_{1} & \cdots & \mu_{n-1} \\
\mu_{1} & \mu_{2} & \cdots & \mu_{n} \\
\vdots & \vdots & \vdots & \vdots \\
\mu_{n-1} & \mu_{n} & \cdots & \mu_{2 n-2}
\end{array}\right), \\
\Delta_{n}=\operatorname{det}\left(M_{n}\right), \quad \Delta_{0}=1 .
\end{gathered}
$$

LEMMA 4.5. Let $A_{i j}$ be the determinant of the matrix obtained from $M_{n}$ by deleting row $i$ and column $j$. If $n=1$ we set $A_{11}=1$. Then

$$
\begin{aligned}
\Delta_{n+1}= & \mu_{n} \sum_{k=1}^{n}(-1)^{k} \mu_{n-1+k} A_{1 k} \\
& +\mu_{n+1} \sum_{k=1}^{n}(-1)^{k+1} \mu_{n-1+k} A_{2 k}+\cdots \\
& +\mu_{n+r} \sum_{k=1}^{n}(-1)^{r+k} \mu_{n-1+k} A_{r+1+k}+\cdots \\
& +\mu_{2 n-1} \sum_{k=1}^{n}(-1)^{n-1+k} \mu_{n-1+k} A_{n k}+\mu_{2 n} \Delta_{n}
\end{aligned}
$$

Proof. The proof follows by induction on $n$.

5. Positive definite sequences. In this section we use the concept of positive definite sequences given by Aheizer and Krein [1] to prove that the sequence of the moments of the spectral function $\tau(\lambda)$ is a positive definite sequence. 
Definition 5.1. Let $J=(a, b)(-\infty \leq a<b \leq \infty)$ be an interval in $\mathbb{R}$. An infinite sequence $\left\{s_{k}\right\}_{k \geq 0}$ is said to be a positive definite sequence on $J$ if for every nonnegative polynomial $R_{n}(\lambda)=p_{0}+p_{1} \lambda+p_{2} \lambda^{2}+\cdots+p_{n} \lambda^{n}$ in $J$, the sequence $\left\{s_{k}\right\}_{k \geq 0}$ satisfies the property

$$
\sum_{j=0}^{n} p_{j} s_{j}>0, \quad \text { for } n=0,1,2, \ldots
$$

THEOREM 5.2. Let $\left\{r_{n}\right\}_{n \geq 1}$ be a sequence of positive real numbers and let $\xi_{1}<\xi_{2}<$ .. be a sequence of real numbers such that $\sum r_{i} \xi_{i}^{k}<\infty$, for all $k \geq 0$. Put

$$
s_{k}=\sum_{i=1}^{\infty} r_{i} \xi_{i}^{k}, \quad k=0,1, \ldots
$$

Then, the sequence $\left\{s_{k}\right\}_{k \geq 0}$ is positive definite in every interval $(a, b)$ satisfying $-\infty \leq$ $a<\xi_{1}<\xi_{2}<\cdots<b \leq \infty$.

Proof. Let $\varphi(\lambda)$ be any real nonnegative polynomial in the interval $(a, b)$, say, $\varphi(\lambda)=\sum_{0}^{n} p_{k} \lambda^{k}$. We have

$$
\sum_{k=0}^{n} p_{k} s_{k}=\sum_{k=0}^{n} \sum_{i=1}^{\infty} p_{k} r_{i} \xi_{i}^{k}=\sum_{i=1}^{\infty} r_{i}\left(\sum_{k=0}^{n} p_{k} \xi_{i}^{k}\right)=\sum_{i=1}^{\infty} r_{i} \varphi\left(\xi_{i}\right)>0 .
$$

Thus the proof is complete.

COROLlary 5.3. Let $\left\{\lambda_{i}\right\}_{i \geq 0}$ and $\left\{\rho_{i}\right\}_{i \geq 0}$ be the eigenvalues and the normalization constants, respectively. If $\lambda_{i} \rho_{i}>0$ for $i \geq 0$, then $\left\{\mu_{i}\right\}_{i \geq 1}$ is a positive definite sequence.

Proof. We define the real numbers

$$
r_{k}=\frac{\lambda_{k-1}}{\rho_{k-1}}, \quad k \geq 1, \xi_{k}=\lambda_{k-1}, k \geq 1 .
$$

If we set

$$
s_{k}=\sum_{1}^{\infty} r_{i} \xi_{i}^{k}, \quad k=0,1, \ldots,
$$

then $r_{k}>0$ for $k \geq 1$. Thus, by Theorem 5.2 , the sequence $\left\{s_{k}\right\}_{k \geq 0}$ is positive definite. By formula (4.3), $s_{k}=\mu_{k+1},(k=0,1, \ldots)$ hence $\left\{\mu_{i}\right\}_{i \geq 1}$ is a positive definite sequence.

DEFINITION 5.4. An infinite real quadratic form

$$
\sum_{i, k=0}^{\infty} a_{i k} \xi_{i} \xi_{k}, \quad\left(a_{i k}=a_{k i}\right)
$$

is said to be positive if all its partial sums

$$
\sum_{i, k=0}^{n} a_{i k} \xi_{i} \xi_{k}, \quad(n=0,1, \ldots)
$$

are positive. 
TheOREM 5.5. The sequence $\left\{s_{n}\right\}_{n \geq 0}$ is positive definite in the interval $(-\infty, \infty)$ if the infinite quadratic form

$$
\sum_{i, k=0}^{\infty} s_{i+k} \xi_{i} \xi_{k}
$$

is positive.

Proof. See [1, page 3, Theorem 1].

THEOREM 5.6. Let $\left\{\lambda_{i}\right\}_{i \geq 0}$ and $\left\{\rho_{i}\right\}_{i \geq 0}$ be the eigenvalues and the normalization constants of Problem 4.1. Let $\lambda_{i} \rho_{i}>0$, and let $\left\{\mu_{i}\right\}_{i \geq 0}$ be a sequence of moments given by (4.4). Then the quadratic form

$$
\sum_{i, k=0}^{\infty} \mu_{i+k+1} \xi_{i} \xi_{k}
$$

is positive.

Proof. Since $\lambda_{i} \rho_{i}>0$, by Corollary 5.3 , the sequence $\{\mu\}_{i \geq 1}$ is positive definite. By Theorem 5.5, the infinite quadratic form

$$
\sum_{i, k=0}^{\infty} \mu_{i+k+1} \xi_{i} \xi_{k}
$$

is positive.

THEOREM 5.7. Let $\left\{\lambda_{i}\right\}_{i \geq 0}$ and $\left\{\rho_{i}\right\}_{i \geq 0}$ be the eigenvalues and the normalization constants of Problem 4.1. Let $\lambda_{i} \rho_{i}>0$, and let $\left\{\mu_{i}\right\}_{i \geq 0}$ be a sequence of moments given by (4.4). Then

$$
\operatorname{det}\left(\begin{array}{cccc}
\mu_{1} & \mu_{2} & \cdots & \mu_{n} \\
\mu_{2} & \mu_{3} & \cdots & \mu_{n+1} \\
\vdots & \vdots & \vdots & \vdots \\
\mu_{n} & \mu_{n+1} & \cdots & \mu_{2 n-1}
\end{array}\right)>0
$$

for $n=1,2, \ldots$

Proof. By Theorem 5.6, the quadratic form

$$
\sum_{i, k=0}^{\infty} \mu_{i+k+1} \xi_{i} \xi_{k}
$$

is positive. Then, the determinants of the principal submatrices are positive, that is,

$$
\operatorname{det}\left(\begin{array}{cccc}
\mu_{1} & \mu_{2} & \cdots & \mu_{n} \\
\mu_{2} & \mu_{3} & \cdots & \mu_{n+1} \\
\vdots & \vdots & \vdots & \vdots \\
\mu_{n} & \mu_{n+1} & \cdots & \mu_{2 n-1}
\end{array}\right)>0
$$

for $n=1,2, \ldots$. 
6. Construction of a solution for Problem 4.1. In this section, we give an explicit solution for Problem 4.1, if the limit circle case holds for the recurrence relation (2.1).

THEOREM 6.1. Let $\tau(\lambda)$ be the spectral function defined by (3.6), and let $B=$ $\operatorname{diag}\left(b_{0}, b_{1}, \ldots\right)$ be given real matrix, where $\left\{b_{n}\right\}_{n \geq 0}$ is a sequence of nonzero real numbers. If the relation

$$
b_{n} \Delta_{n} \Delta_{n+1}>0
$$

holds for all $n \geq 0$, then there exists a countable set of orthogonal polynomials $\left\{y_{n}(\lambda)\right\}_{n \geq 0}$ in the sense of dual orthogonality property (3.13), and the polynomials are determined up to change of sign. Moreover, the polynomials $\left\{y_{n}(\lambda)\right\}_{n \geq 0}$ are dense in $L_{T}^{2}$.

Proof. For the first part, we seek polynomials of the form

$$
y_{n}(\lambda)=\beta_{n}\left(\lambda^{n}+\sum_{k=0}^{n-1} \alpha_{n k} \lambda^{k}\right), \quad k=0,1, \ldots,
$$

where $y_{0}(\lambda)=\beta_{0}$ and $\beta_{n} \neq 0$. Using (3.13) and (4.3) we get

$$
\beta_{0}^{2}=\frac{1}{\mu_{0} b_{0}}=\frac{\Delta_{0}}{b_{0} \Delta_{1}}
$$

that is positive by assumption. It follows from (6.2) that

$$
\left(y_{n}(\lambda)\right)^{2}=\beta_{n}^{2}\left[\lambda^{2 n}+\sum_{k=0}^{n-1} \alpha_{n k} \lambda^{n+k}\right]+\beta_{n} y_{n}(\lambda) \sum_{k=0}^{n-1} \alpha_{n k} \lambda^{k}, \quad n=1,2, \ldots
$$

Combining (3.13), (4.2), and (6.4), it follows that

$$
b_{n}^{-1}=\beta_{n}^{2}\left(\mu_{2 n}+\sum_{k=0}^{n-1} \alpha_{n k} \mu_{n+k}\right)
$$

This gives $\beta_{n}$ in terms of $\alpha_{n k}$ and the moments. To determine $\alpha_{n k}$, we substitute $y_{n}(\lambda)$ given by (6.2) in (4.2) and we obtain

$$
\mu_{n+k}+\sum_{k=0}^{n-1} \alpha_{n k} \mu_{n+k}=0
$$

where $0 \leq k \leq n-1$ and $n=1,2, \ldots$ Using matrix notation, we have

$$
M_{n}\left(\alpha_{n 0}, \alpha_{n 1}, \ldots, \alpha_{n, n-1}\right)^{T}=\left(-\mu_{n},-\mu_{n+1}, \ldots,-\mu_{2 n-1}\right)^{T}
$$


Therefore

$$
\alpha_{n r}=\frac{\sum_{k=1}^{n}(-1)^{k+r} \mu_{n-1+k} A_{r+1, k}}{\Delta_{n}}
$$

where $0 \leq r \leq n-1$ and $n=1,2, \ldots$ Substituting (4.6) in (6.8), we get

$$
\Delta_{n+1}=\Delta_{n}\left(\mu_{2 n}+\sum_{k=0}^{n-1} \alpha_{n k} \mu_{n+k}\right)
$$

Using (6.5) in (6.9) we obtain

$$
\beta_{n}^{2}=\frac{\Delta_{n}}{\Delta_{n+1} b_{n}}
$$

that is positive by the assumption, and this completes the proof. For the second part see [2, page 141].

THEOREM 6.2. Let the assumptions of Theorem 6.1 hold. Then, the Jacobi matrix $A$ of the form

$$
A=\left(\begin{array}{cccccc}
a_{0} & c_{0} & 0 & \ldots & \ldots & \ldots \\
c_{0} & a_{1} & c_{1} & 0 & \ldots & \ldots \\
0 & c_{1} & a_{2} & c_{2} & 0 & \ldots \\
\vdots & \vdots & \vdots & \vdots & \vdots & \vdots
\end{array}\right)
$$

given by

$$
\begin{gathered}
a_{0}=b_{0} \alpha_{1,0}, \quad a_{n}=b_{n}\left(\alpha_{n, n-1}-\alpha_{n+1, n}\right), \quad n=1,2, \ldots, \\
\alpha_{n r}=\frac{\sum_{k=1}^{n}(-1)^{k+r} \mu_{n-1+k} A_{r+1, k}}{\Delta_{n}}, \quad 0 \leq r \leq n-1, n=1,2, \ldots, \\
c_{n}=\frac{b_{n} \beta_{n}}{\beta_{n+1}}, \quad \beta_{n}=\frac{\Delta_{n}}{\Delta_{n+1} b_{n}}, \quad n=0,1, \ldots,
\end{gathered}
$$

is a solution for Problem 4.1, which assumes $\tau(\lambda)$ as its spectral function, where

$$
y(\lambda)=\left(y_{0}(\lambda), y_{1}(\lambda), \ldots\right)^{T}, \quad y_{n}(\lambda)=\beta_{n}\left(\lambda^{n}+\sum_{r=0}^{n-1} \alpha_{n r} \lambda^{r}\right), \quad n=0,1, \ldots
$$

Moreover, the matrix A is positive definite in the sense of Definition 5.4.

Proof. Substituting $y_{n}(\lambda)$ given by (6.2) in the recurrence relation (2.1) and comparing the corresponding coefficients of the powers of $\lambda$, we obtain

$$
\begin{gathered}
a_{0}=-b_{0} \alpha_{1,0}, \quad c_{n} \beta_{n+1}=b_{n} \beta_{n}, \\
c_{n} \beta_{n+1} \alpha_{n+1, n}+a_{n} \beta_{n}=b_{n} \beta_{n} \alpha_{n, n-1}, \quad n \geq 1 .
\end{gathered}
$$


By substituting (6.14) in (6.15) we get

$$
a_{n}=b_{n}\left(\alpha_{n, n-1}-\alpha_{n+1, n}\right),
$$

which completes the first part of the proof. In order to prove $A$ is positive definite, by Definition 5.4, it suffices to prove that the determinants of leading principal submatrices of $A$ are all positive. Let $D_{n}$ be the determinant of the $n \times n$ leading submatrix of $A$ in the upper left corner of $A$. It is easy to check by induction that

$$
D_{n}=b_{0} b_{1} \cdots b_{n-1}(-1)^{n} \alpha_{n, 0} .
$$

This is equivalent to

$$
D_{n}=\frac{b_{0} \triangle_{0}}{\triangle_{1}} \frac{b_{1} \triangle_{1}}{\triangle_{2}} \cdots \frac{b_{n-1} \triangle_{n-1}}{\triangle_{n}}(-1)^{n} \alpha_{n, 0} \triangle_{n}
$$

By using (6.8) we obtain

$$
(-1)^{n} \alpha_{n, 0} \triangle_{n}=\operatorname{det}\left(\begin{array}{cccc}
\mu_{1} & \mu_{2} & \cdots & \mu_{n} \\
\mu_{2} & \mu_{3} & \cdots & \mu_{n+1} \\
\vdots & \vdots & \vdots & \vdots \\
\mu_{n} & \mu_{n+1} & \cdots & \mu_{2 n-1}
\end{array}\right)
$$

Therefore, combining (6.19) with the assumption of Theorem 6.1 implies that $D_{n}>0$ if and only if

$$
\operatorname{det}\left(\begin{array}{cccc}
\mu_{1} & \mu_{2} & \cdots & \mu_{n} \\
\mu_{2} & \mu_{3} & \cdots & \mu_{n+1} \\
\vdots & \vdots & \vdots & \vdots \\
\mu_{n} & \mu_{n+1} & \cdots & \mu_{2 n-1}
\end{array}\right)>0
$$

which is true by Theorem 5.7, for $n=1,2, \ldots$ This completes the proof.

ACKNOWLEDGEMENTS. The author would like to thank Professor Angelo Mingarelli for his support, and the anonymous referee for valuable comments. The author is also indebted to the Ministry of Culture and Higher Education of Islamic Republic of Iran for the financial support of this work.

\section{REFERENCES}

[1] N. I. Ahiezer and M. Krein, Some Questions in the Theory of Moments, Translations of Mathematical Monographs, vol. 2, American Mathematical Society, Rhode Island, 1962. MR 29\#5073. Zbl 117.32702.

[2] F. V. Atkinson, Discrete and Continuous Boundary Problems, Mathematics in Science and Engineering, vol. 8, Academic Press, New York, 1964. MR 31\#416. Zbl 117.05806.

[3] K. Ghanbari and A. Mingarelli, Generalized inverse eigenvalue problem for symmetric matrices, Int. J. Appl. Math. 4 (2000), no. 2, 199-209.

[4] O. H. Hald, Inverse eigenvalue problems for Jacobi matrices, Linear Algebra and Appl. 14 (1976), no. 1, 63-85. MR 56\#5594. Zbl 328.15007. 
[5] J. Janas and S. Naboko, Jacobi matrices with absolutely continuous spectrum, Proc. Amer. Math. Soc. 127 (1999), no. 3, 791-800. MR 99f:47042. Zbl 910.47022.

Kazem Ghanbari: School of Mathematics and Statistics, Carleton University, OTTAWA, CANADA ON K1S 5B6

E-mail address: kghanbar@math. carleton.ca 


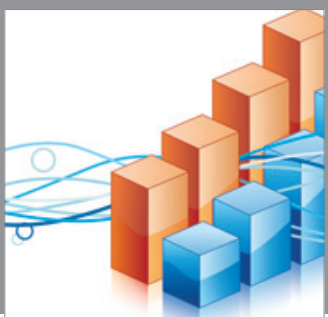

Advances in

Operations Research

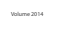

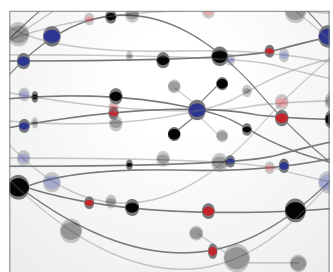

\section{The Scientific} World Journal
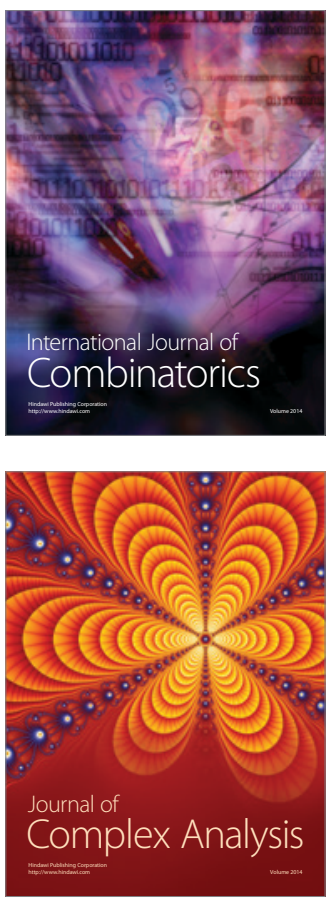

International Journal of

Mathematics and

Mathematical

Sciences
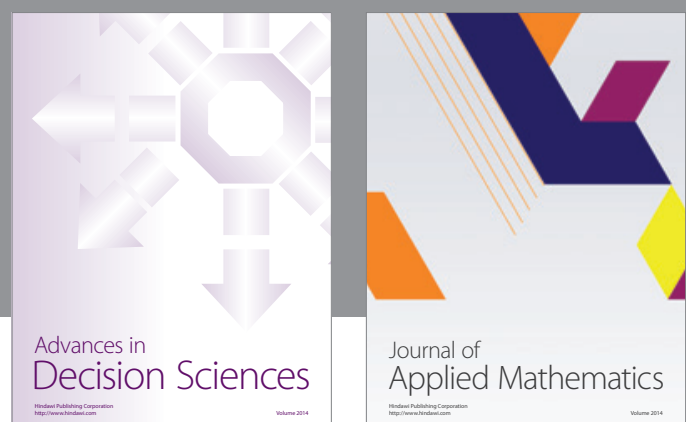

Journal of

Applied Mathematics
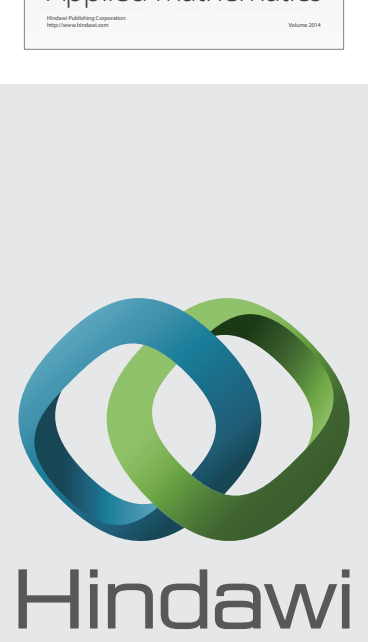

Submit your manuscripts at http://www.hindawi.com
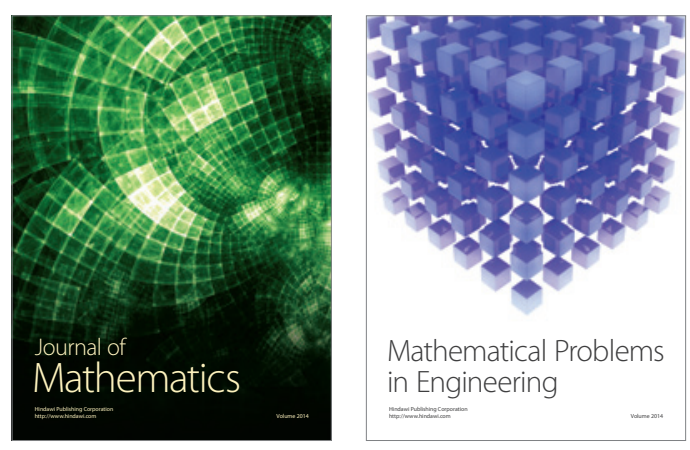

Mathematical Problems in Engineering
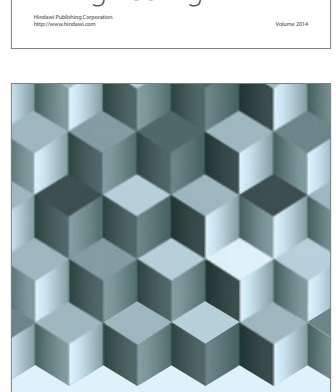

Journal of

Function Spaces
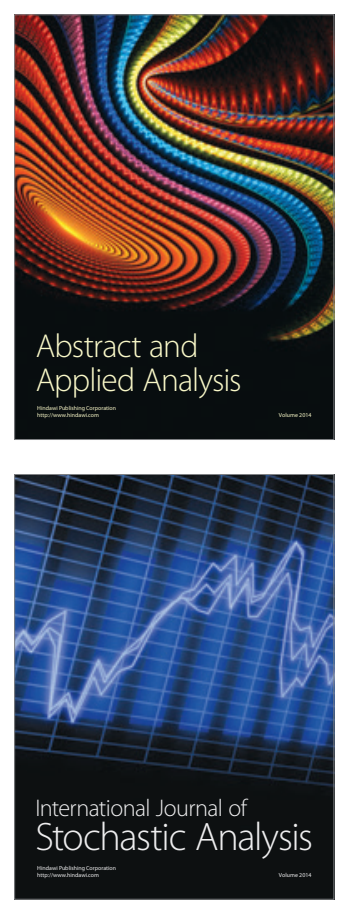

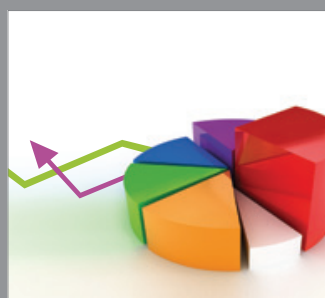

ournal of

Probability and Statistics

Promensencen
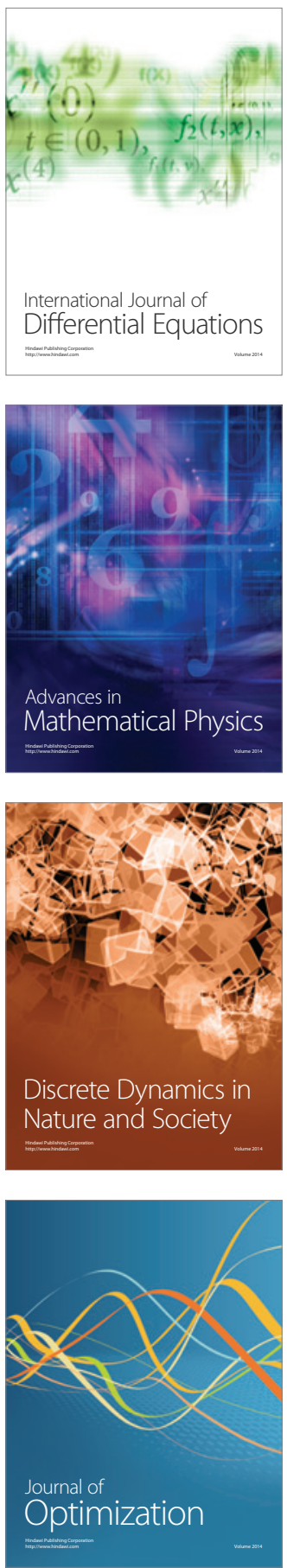